\title{
Surface properties of icy transneptunian objects from the second ESO large program
}

\author{
Francesca E. DeMeo ${ }^{1}$, Maria Antonietta Barucci ${ }^{1}$, Alvaro \\ Alvarez-Candal ${ }^{2}$, Catherine de Bergh ${ }^{1}$, Sonia Fornasier ${ }^{1,3}$, Frédéric \\ Merlin $^{4}$, Davide Perna ${ }^{1,5,6}$, and Irina Belskaya ${ }^{1,7}$ \\ ${ }^{1}$ LESIA, Observatoire de Paris, 5, Place Jules Janssen, 92195 Meudon Cedex, France \\ email: francesca.demeo@obspm.fr \\ ${ }^{2}$ European Southern Observatory, Alonso de Cordova 3107, Santiago, Chile \\ ${ }^{3}$ Université de Paris 7 Denis Diderot, Paris, France \\ ${ }^{4}$ Department of Astronomy, University of Maryland, College Park, MD 20742, USA \\ ${ }^{5}$ INAF- Osservatorio Astronomico di Roma, Via Frascati 33, I-00040 \\ Monte Porzio Catone, Italy \\ ${ }^{6}$ Università di Roma Tor Vergata, Via della Ricerca Scientifica 1, I-00133 Roma, Italy \\ ${ }^{7}$ Institute of Astronomy, Kharkiv National University, 35 Sumska str., 61022 Kharkiv, Ukraine
}

\begin{abstract}
An analysis is well underway for the data from the second Large Program (PI M. A. Barucci) dedicated to investigating the surface properties of Centaurs and Transneptunian objects through spectroscopic, photometric color, lightcurve, and polarimetric observations using the European Southern Observatory (ESO) Very Large Telescope (VLT) and New Technology Telescope (NTT). 45 objects were observed between 2006 and 2008, allowing a broad characterization of at least the largest and brightest objects among this population. In this report, we summarize all our findings, but focus on the analysis of the presence of ices such as methane, ethane, nitrogen, ammonia hydrate, methanol, and particularly $\mathrm{H}_{2} \mathrm{O}$ which is so abundant throughout the outer solar system.
\end{abstract}

Keywords. Kuiper Belt, techniques: spectroscopic, techniques: photometric, techniques: polarimetric

\section{Introduction}

The Transneptunian and Centaur populations display a wide variety of characteristics. While all are considered small bodies, they range from tens to thousands of kilometers in diameter with densities from less than one for many small bodies to greater than $2 \mathrm{~g} / \mathrm{cm}^{3}$. Their spectra, revealing information about surface composition, have visible slopes ranging from nearly neutral to the reddest in the solar system. Near-infrared spectra of some objects have diagnostic absorption bands that can often be clearly linked to ices. TNO spectra are typically divided into several compositional groups. Featureless spectra have a wide range of slopes but no distinct absorption bands, thus leaving the surface composition largely unknown. $\mathrm{H}_{2} \mathrm{O}$ ice rich spectra are abundant in the outer solar system (Barkume et al. 2008; Guilbert et al. 2009a). There are large differences in the strengths of the $\mathrm{H}_{2} \mathrm{O}$ bands among bodies, where the strongest are seen on (136108) Haumea and its collisional family (Brown et al. 2007). All high signal-to-noise spectra of $\mathrm{H}_{2} \mathrm{O}$-rich spectra display the 1.65 - $\mu$ m feature suggesting the crystalline phase, although the feature is not detected on smaller objects with admittedly lower quality spectra. Other ices are found only rarely. Methanol has been detected on two TNOs ((5145) Pholus and $\left.2002 \mathrm{VE}_{95}\right), \mathrm{NH}_{3}$ on Orcus and Pluto's satellite Charon, and methane on Pluto, Eris, Quoaoar, and Sedna. This diverse population has been under scrutiny since 
the first TNO discovery in 1992 since Pluto to understand its formation, evolution, and present state as well as what it can tell us about the solar system as a whole.

\section{Overview of Large Program Results}

A second Large Program (PI M. A. Barucci) dedicated to investigating the surface properties of Centaurs and Transneptunian objects was carried out between 2006 and 2008 using the European Southern Observatory (ESO) Very Large Telescope (VLT) and New Technology Telescope (NTT). Observational methods included spectroscopy, photometry (colors and lightcurves), and polarimetry. This variety of techniques was chosen to obtain complementary information about each body that any single method could not provide. Here we summarize the current state of the analysis of the 45 objects that were observed, and focus specifically on the presence of ices detected by features in near-infrared spectra.

\subsection{Polarimetric Measurements}

A behavior of linear polarization degree versus phase angle strongly depends on top surface layer properties, such as albedo, complex refractive index, particle size, packing density, and microscopic optical heterogeneity. 8 TNOs and Centaurs were observed: (136199) Eris, (136108) Haumea, (20000) Varuna, (38628) Huya, 26375 (1999 DE9), (2060) Chiron, (5145) Pholus and (10199) Chariklo. Negative polarization was measured for all objects observed, varying from $-0.3 \%$ to $-2 \%$. For TNOs two different behaviors of polarization phase dependencies were discovered (Bagnulo et al. 2008). Objects with a diameter smaller than $1000 \mathrm{~km}$ exhibit a negative polarization that rapidly increases (in absolute value) with the phase angle and reaches about $1 \%$ with a phase angle as small as 1 degree. The largest TNOs exhibit a small fraction of negative linear polarization which does not noticeably change in the observed phase angle range. Different polarimetric behaviors of these two groups, which indicate considerable differences in their surface properties, are probably related to the retention of volatiles (Bagnulo et al. 2008). The modeling of polarimetric behavior of the largest objects suggests that their topmost surface layer consists of large (compared to wavelength) inhomogeneous particles (Belskaya et al. 2008). Smaller size TNOs characterized by a pronounced branch of negative polarization revealed similar polarization behavior regardless of the fact that they have different surface albedos and belong to different dynamical groups. On the other hand, all three Centaurs observed so far show different polarization phase angle behaviors (Belskaya et al. 2009). It implies greater diversity in surface characteristics of Centaurs compared to that of TNOs.

\subsection{Lightcurves}

Measuring the lightcurves of TNOs enables the calculation of the rotational period when there is a significant periodic amplitude. Lower limits to the axis ratio a/b of an ellipsoid that could represent the body can also be found by assuming all magnitude changes are due to an elongated shape rather than albedo variations. One can also constrain the lower limits on the density based on the synodic periods and lightcurve amplitudes, which can provide important constraints on the internal structure of TNOs. Rotational periods also help reveal the collisional history of TNOs since the smaller bodies are expected to be collisional products of originally larger bodies.

There were about 40 TNOs and Centaurs that had determined rotational periods before this Large Program. 7 new objects were observed by Dotto et al. (2008) and Perna et al. (2009a). Dotto et al. (2008) measured 3 new objects ((65489) Ceto, (90568) $2004 \mathrm{GV}_{9}$, 
and (95626) $2002 \mathrm{GZ}_{32}$ ), and find quite short rotational periods and densities low enough to put these bodies within the limits for rotationally stable ellipsoids. Perna et al. (2009a) calculate 4 new rotational periods ((144897) $2004 \mathrm{UX}_{10}$, (145451) $2005 \mathrm{RM}_{43}$, (145453) $2005 \mathrm{RR}_{43}$, and $\left.2003 \mathrm{UZ}_{413}\right) .2003 \mathrm{UZ}_{413}$ is found to be a quite peculiar rapidly rotating object with a high estimated density. The results from these works have put into question the size-density trend found by Sheppard et al. (2008). While brighter objects are more dense, it now appears that fainter objects have a wider range of densities than previously thought (Perna et al. 2009a), which indicates the importance of improving TNO density statistics and albedo measurements in order to assess the existence of any size-density relationship.

\subsection{Photometric Colors}

Measuring photometric colors is the best methods of characterizing the surfaces of a large sample of TNOs. While spectroscopy provides more resolution, photometric measurements are often the only possibility for dimmer (smaller, darker, and/or farther) objects. Colors of TNOs and Centaurs in the visible and near-infrared were reported by DeMeo et al. (2009) and Perna et al. (2009b) for 45 objects, 19 of which had no previous photometric color measurements. TNOs and Centaurs are divided into four taxonomic groups, BB, BR, IR, and RR that span from neutral to very red.

Our measurements of three objects could not be classified in this system (26375, 145452, and $2007 \mathrm{UK}_{126}$ ), because their visible colors were red, matching best the IR and RR classes, while the near-infrared colors were bluer, consistent with the BB and BR classes. This suggests greater color diversity than what was found in the original sample used to create the taxonomy. In analysis of the distribution of taxonomic classes among dynamical classes and orbital elements, it was found that 8 of the 13 Centaurs in the sample were in the neutral to moderately red BB and BR classes while the other 5 were in the RR class, confirming the bimodality of the population (Peixinho et al. 2003). All IR class objects in our sample are classical objects, which is consistent with Fulchignoni et al. (2008) where they found the IR class confined to the resonant and classical population.

\subsection{Spectral Measurements - Featureless and $\mathrm{H}_{2} \mathrm{O}$-rich bodies}

Visible spectra for 43 TNOs and Centaurs were measured in Alvarez-Candal et al. (2008) and Fornasier et al. (2009), and 22 of these had no previously published spectra. Both authors find most spectra to be featureless. Alvarez-Candal et al. (2008) find that (208996) $2003 \mathrm{AZ}_{84}$, (10199) Chariklo, and (42355) Typhon have a broad, shallow feature centered at $0.65 \mu \mathrm{m}$ for the first two and $0.6 \mu \mathrm{m}$ for the latter. A similar band is seen on dark asteroids, and has been thought to be be due to the presence of aqueously altered minerals (Vilas et al. 1994), however, it is unknown how temperatures in the outer solar system could be high enough for aqueous alteration to occur.

Observations of (60558) Echeclus by Alvarez-Candal et al. (2008) had a visible slope $50 \%$ less than previous measurements. They also find that many spectra tend to flatten out past $0.75 \mu \mathrm{m}$ relative to the visible slope. Observations of (42355) Typhon by AlvarezCandal et al. (2009) confirm the previously identified feature, however, the spectrum of (208996) $2003 \mathrm{AZ}_{84}$ did not have the weak 0.65 - $\mu$ m feature seen originally by AlvarezCandal et al. (2008), suggesting surface heterogeneity. The visible slopes of 73 objects from these works and the literature were collected by Fornasier et al. (2009) and the mean was found to be $17.9 \% / 10^{3} \AA$. The classical TNOs had a deficit of very red slopes compared with other populations with no slopes exceeding $35 \% / 10^{3} \AA$, although this is likely an observational bias because the redder objects tend to be smaller. They also find 
that the TNO/Centaur population has a wider distribution of slopes than the Trojan asteroids that may have originated in the Kuiper Belt.

21 near-infrared spectra were presented in Guilbert et al. (2009a). Of this sample, 4 are featureless, 6 show clear signatures of $\mathrm{H}_{2} \mathrm{O}$ ice, 7 show more uncertain signatures of $\mathrm{H}_{2} \mathrm{O}$ ice, 1 (Eris) has methane, and the signal-to-noise ratio of the last three is too low to analyze. The objects with rare spectral features are further discussed in the next section. Plutino 47171 was modeled by Protopapa et al. (2009) using Titan tholin, Triton tholin diluted in $\mathrm{H}_{2} \mathrm{O}$ and either amorphous carbon or serpentine. Their spectrum differs slightly from two previously published spectra, suggesting surface heterogeneity. Alvarez-Candal et al. (2009) found that the spectrum of the scattered disk object (42355) Typhon could overall be well modeled with Titan and Triton tholins, $\mathrm{H}_{2} \mathrm{O}$ ice, amorphous carbon, and serpentine. What could not be modeled, however, is the broad, shallow band around 0.6 $\mu \mathrm{m}$. Merlin et al. (2009b) model 6 TNOs and find that the surfaces have $\mathrm{H}_{2} \mathrm{O}$ ice and a dark material that is likely an irradiated product. They find objects could have both crystalline and amorphous $\mathrm{H}_{2} \mathrm{O}$ ice on the surface, which is consistent with the balance between the states at low temperatures seen in the laboratory results of Zheng et al. (2008). However, the laboratory samples only undergo partial irradiation (e- only) and so other higher energetic irradiation sources must still be considered.

10199 Chariklo is a Centaur with a diameter of around 260 kilometers and an albedo of approximately $5.7 \%$ (Stansberry et al. 2008). Chariklo has been observed six times since 1997 and, interestingly, the first spectra display significant $\mathrm{H}_{2} \mathrm{O}$ ice features, while the most recent from Guilbert et al. (2009a,b) are nearly featureless with insignificant amounts of $\mathrm{H}_{2} \mathrm{O}$. Since the rotational period of Chariklo is not known, it is unclear whether these differences are due to surface heterogeneity or if it is a temporal change (Guilbert et al. 2009b). Chariklo orbits the sun between 13 and 18.5 AU, so the spectral change is not likely due to its approach toward the sun because the distance change is not so great. Additionally, Guilbert et al. (2009b) report that no cometary signatures were found from recent images of Chariklo. In models of the spectra they show that Chariklo could be comprised of an irradiated coating and an underlying fresher layer that has been partially resurfaced by impacts.

\subsection{Spectral Measurements - Large Icy Bodies}

Three of the bodies we observed, which were also among the largest, have more unique spectral features. (90482) Orcus, (50000) Quaoar, and (136199) Eris have other ices besides $\mathrm{H}_{2} \mathrm{O}$ on their surface. The results of the analysis of each body is presented here.

Barucci et al. (2008) observed the TNO (90482) Orcus in January of 2007 and present the best quality data to-date of this $\mathrm{H}_{2} \mathrm{O}$-rich object. Orcus shows deep $\mathrm{H}_{2} \mathrm{O}$ features at 1.5 and $2 \mu \mathrm{m}$ as well the 1.65 feature indicating crystallinity. They also detected a feature with a depth of $10 \%$ at $2.216 \mu \mathrm{m}$ that resembles the ammonia hydrate feature seen on Charon. Barucci et al. (2008) model the spectrum using amorphous and crystalline $\mathrm{H}_{2} \mathrm{O}$ ice, ammonia diluted in crystalline water ice and a blue component artificially created to reproduce the negative overall slope of Orcus' spectrum. It is difficult to firmly identify the $\sim 2.2-\mu \mathrm{m}$ feature as ammonia hydrate based on one weak band. Further studies of objects with this band, however, are important because $\mathrm{NH}_{3}$ is expected to be quickly destroyed by irradiation (Strazzulla and Palumbo 1998; Cooper et al. 2003). Proposed mechanisms to resupply $\mathrm{NH}_{3}$ to the surface include cryovolcanism, impact gardening, and solid-state greenhouse or convection, with cryovolcanism being the favored mechanism (Cook et al. 2007). These phenomena could also explain the abundance of crystalline $\mathrm{H}_{2} \mathrm{O}$ ice among TNOs, whose presence is intriguing because it should be amorphized by space weathering over the age of the solar system. 
(50000) Quaoar has a diameter of $\sim 840 \mathrm{~km}$ and is a relatively bright object among TNOs with an albedo of $19 \%$ (Stansberry et al. 2008). The visible spectrum is very red, with a slope of about 28\% per $100 \mathrm{~nm}$ (Alvarez-Candal et al. 2008). Quaoar's nearinfrared spectrum is unique because, although it displays clear $\mathrm{H}_{2} \mathrm{O}$ features at 1.5, 1.65, and $2 \mu \mathrm{m}$ and another feature near $2.2 \mu \mathrm{m}$ as seen on Charon and Orcus, there are other weak features present that suggest the $2.2-\mu \mathrm{m}$ feature is due to methane rather than $\mathrm{NH}_{3}$. Schaller and Brown (2007a) first suggested the presence of methane on Quaoar's surface while simultaneously detecting weak features that match those of ethane in the spectrum. Quaoar is a transition object that has not undergone complete volatile loss (Schaller and Brown 2007b). Dalle Ore et al. (2009) model a spectrum from the visible to near-infrared including the additional constraints of Spitzer data at 3.6 and $4.5 \mu \mathrm{m}$ and find a best model consisting of an intimate mixture of crystalline and amorphous $\mathrm{H}_{2} \mathrm{O}$, $\mathrm{CH}_{4}, \mathrm{~N}_{2}$, and $\mathrm{C}_{2} \mathrm{H}_{6}$ ices with Triton and Titan tholins. They find that amorphous $\mathrm{H}_{2} \mathrm{O}$ ice increases the albedo at the wavelengths of the second Spitzer band, improving the fit, but the model still underestimates the reflectance, suggesting that either the quality of the optical constants used is not sufficient, or that there is another component on the surface missing in the model.

Merlin et al. (2009a) present two spectra of the largest TNO (136199) Eris, whose diameter is $\sim 2400 \mathrm{~km}$ (Brown et al. 2006). Eris' spectrum in the near-infrared is dominated by deep and broad methane features suggesting large grain sizes. A detailed analysis of the spectra by Merlin et al. (2009a) reveals that there are small wavelength shifts in the position of methane bands compared to those obtained from laboratory measurements of pure methane ice. They propose several possible reasons for this shift: geographic versus intimate mixing of material, hydrated and diluted materials, temperature and physical state, and irradiation effects. Dilution of methane in $\mathrm{N}_{2}$ is the most likely cause of the shift, even though $\mathrm{N}_{2}$ cannot be directly recognized since it is probably in its alpha state (below $35.6 \mathrm{~K}$ ) which has a very narrow band in the near-infrared that is not detectable at the spectral resolution of the data. The amount of nitrogen on Eris is expected to be much smaller than on Pluto and Triton because of the stronger $1.689-\mu \mathrm{m}$ pure methane feature and the less significant wavelength shifts. The deepest bands in in the visible wavelengths of Eris' spectrum are more shifted than the weakest suggesting a stratification of diluted methane ice.

\section{Conclusions}

The second ESO Large Program devoted to characterizing TNOs has contributed immensely to our understanding of these bodies by using many observational techniques. Advancement of the discovery and analysis of ices on TNO surfaces has been achieved particularly through near-infrared spectral measurements where diagnostic ice signatures are present. The presence or absence of these features has been determined for the 45 objects in this study, although more detailed modeling and analysis of about 20 objects in the sample is still underway. We await the final results from this program, as well as many more interesting discoveries and breakthroughs through the coming years and decades aided by discovery surveys and improvements in technology.

\section{References}

Alvarez-Candal, A., Barucci, M. A., Merlin, F., de Bergh, C., Fornasier, S., Guilbert, A., \& Protopapa, S. 2009, A\&BA, submitted

Alvarez-Candal, A., Fornasier, S., Barucci, M. A., de Bergh, C., \& Merlin, F. 2008, A\&\&A, 487, 741 
Bagnulo, S., Belskaya, I., Muinonen, K., Tozzi, G. P., Barucci, M. A., Kolokolova, L., \& Fornasier, S. $2008, A \& A$, 491, L33

Barkume, K. M., Brown, M. E., \& Schaller, E. L. 2008, AJ, 135, 55

Barucci, M. A., Merlin, F., Guilbert, A., de Bergh, C., Alvarez-Candal, A., Hainaut, O., Doressoundiram, A., Dumas, C., Owen, T., \& Coradini, A. 2008, A\& A, 479, L13

Belskaya, I., Bagnulo, S., Muinonen, K., Barucci, M. A., Tozzi, G. P., Fornasier, S., \& Kolokolova, L. $2008, A \mathscr{E} A, 479,265$

Belskaya, I., Bagnulo, S., Barucci, M. A., Muinonen, K., Tozzi, G. P., Fornasier, S., \& Kolokolova, L. 2009, Icarus, submitted

Brown, M. E., Barkume, K. M., Ragozzine, D., Schaller, E. L. 2007, Nature, 446, 294

Brown, M. E., Schaller, E. L., Roe, H. G., Rabinowitz, D. L., \& Trujillo, C. A. 2006, ApJ, 643, L61

Cook, J. C., Desch, S. J., Roush, T. L., Trujillo, C. A., \& Geballe, T. R. 2007, ApJ, 663, 1406

Cooper, J. F., Christian, E. R., Richardson, J. D., \& Wang, C. 2003, EMESP, 92, 261

Dalle Ore, C. M., Barucci, M. A., Emery, J. P., Cruikshank, D. P., Dalle Ore, L. V., Merlin, F., Alvarez-Candal, A., de Bergh, C., Trilling, D. E., Perna, D., Fornasier, S., Mastrapa, R. M. E., \& Dotto, E. 2009, A\&\&A, 501, 349

DeMeo, F. E., Fornasier, S., Barucci, M. A., Perna, D., Protopapa, S., Alvarez-Candal, A., Delsanti, A., Doressoundiram, A., Merlin, F., \& de Bergh, C. 2009, A\&̋A, 493, 283

Dotto, E., Perna, D., Barucci, M. A., Rossi, A., de Bergh, C., Doressoundiram, A., \& Fornasier, S. $2008, A \mathscr{E} A, 490,829$

Fornasier, S., Barucci, M. A., de Bergh, C., Alvarez-Candal, A., DeMeo, F. E., Merlin, F., Perna, D., Guilbert, A., Delsanti, A., Dotto, E., \& Doressoundiram, A. 2009, A\&A A, accepted

Fulchignoni, M., Belskaya, I., Barucci, M. A., de Sanctis, M. C., \& Doressoundiram, A. 2008, in: M. A. Barucci, H. Boehnhardt, D. Cruikshank \& A. Morbidelli (eds.), The Solar System Beyond Neptune, (Tucson: Univ. of Arizona Press), p. 181

Guilbert, A., Alvarez-Candal, A., Merlin, F., Barucci, M. A., Dumas, C., de Bergh, C., \& Delsanti, A. 2009a, Icarus, 201, 272

Guilbert, A., Barucci, M. A., Brunetto, R., Delsanti, A., Merlin, F., Alvarez-Candal, A., Fornasier, S., de Bergh, C., \& Sarid, G. 2009b, A\&A, 501, 777

Merlin, F., Alvarez-Candal, A., Delsanti, A., Fornasier, S., Barucci, M. A., DeMeo, F. E., de Bergh, C., Doressoundiram, A., Quirico, E., \& Schmitt, B. 2009a, AJ,137, 315

Merlin, F., Barucci, M. A., de Bergh, C., Fornasier, S., Doressoundiram, A., Perna, D., \& Protopapa, S. 2009b, Icarus, submitted

Peixinho, N., Doressoundiram, A., Delsanti, A., Boehnhardt, H., Barucci, M. A., \& Belskaya, I. 2003, A\& $A, 410$, L29

Perna, D., Dotto, E., Barucci, M. A., Rossi, A., Fornasier, S., \& de Bergh, C. 2009a, A\&A, accepted

Perna, D., Barucci, M. A., Fornasier, S., Demeo, F. E., Alvarez-Candal, A., Merlin, F., Dotto, E., Doressoundiram, A., \& de Bergh, C. 2009b, A\&AA, submitted

Protopapa, S., Alvarez-Candal, A., Barucci, M. A., Tozzi, G. P., Fornasier, S., Delsanti, A., \& Merlin, F. 2009, A\& A, 501, 375

Schaller, E. L. \& Brown, M. E. 2007a, ApJ, 659, L61

Schaller, E. L. \& Brown, M. E. 2007b, ApJ, 670, L49

Sheppard, S. S., Lacerda, P., \& Ortiz, J. L. 2008, in: M. A. Barucci, H. Boehnhardt, D. Cruikshank \& A. Morbidelli (eds.), The Solar System Beyond Neptune, (Tucson: Univ. of Arizona Press), p. 129

Stansberry, J., Grundy, W., Brown, M., Cruikshank, D., Stansberry, J., Grundy, W., Brown, M., Cruikshank, D., Spencer, J., Trilling, D., \& Margot, J. 2008, in: M. A. Barucci, H. Boehnhardt, D. Cruikshank \& A. Morbidelli (eds.), The Solar System Beyond Neptune, (Tucson: Univ. of Arizona Press), p. 161

Strazzulla, G. \& Palumbo, M. E. 1998, P\&SS, 46, 1339

Vilas, F., Jarvis, K. S., \& Gaffey, M. J. 1994, Icarus, 109, 274

Zheng, W., Jewitt, D., \& Kaiser, R. I. 2008, arXiv:0801.2805 